\title{
Prognostic relevance of the mitotic count and the amount of viable tumour after neoadjuvant chemotherapy for primary, localised, high-grade soft tissue sarcoma
}

\begin{abstract}
D Andreou ${ }^{*}, 1,2$, M Werner ${ }^{3}$, D Pink ${ }^{4}$, F Traub ${ }^{2}$, M Schuler ${ }^{5}$, G Gosheger $^{1}$, B Jobke ${ }^{6}$, P Reichardt ${ }^{7}$ and P U Tunn ${ }^{2}$
${ }^{1}$ Department of General Orthopedics and Tumor Orthopedics, Münster University Hospital, Albert-Schweitzer-Campus 1, 48149 Münster, Germany; ${ }^{2}$ Department of Orthopedic Oncology, Sarcoma Center Berlin-Brandenburg, HELIOS Klinikum Berlin-Buch, Schwanebecker Chaussee 50, 13125 Berlin, Germany; ${ }^{3}$ Department of Pathology, Sarcoma Center Berlin-Brandenburg, HELIOS Klinikum Emil von Behring, Walterhöferstraße 11, 14165 Berlin, Germany; ${ }^{4}$ Department of Hematology, Oncology and Palliative Care, Sarcoma Center Berlin-Brandenburg, HELIOS Klinikum Bad Saarow, Pieskower Straße 33, 15526 Bad Saarow, Germany; ${ }^{5}$ Department of Internal Medicine I, University Hospital Carl Gustav Carus Dresden, Fetscherstraße 74, 01307 Dresden, Germany; ${ }^{6}$ Department of Radiology, HELIOS Klinikum Berlin-Buch, Schwanebecker Chaussee 50, 13125 Berlin, Germany and 'Department of Interdisciplinary Oncology, Sarcoma Center Berlin-Brandenburg, HELIOS Klinikum Berlin-Buch, Schwanebecker Chaussee 50, 13125 Berlin, Germany
\end{abstract}

Background: We sought to examine whether mitotic count (MC) and the amount of viable tumour (VT) following neoadjuvant systemic chemotherapy (SC) for primary, localised, high-grade soft tissue sarcoma (STS) correlate with prognosis.

Methods: Retrospective analysis of 57 patients who underwent SC involving a combination of an anthracycline and an alkylating agent, followed by surgical resection between 2001 and 2011.

Results: The amount of VT after chemotherapy was significantly associated with disease-specific survival (DSS) and event-free survival (EFS). Patients with $<10 \%$ VT had a DSS of $94 \%$ at 5 years, compared with $61 \%$ for patients with $\geqslant 10 \%$ VT $(P=0.033)$; EFS was $75 \%$, compared with $48 \%(P=0.030)$. Patients with an $M C$ of $\geqslant 20 / 10$ high power fields (HPF) after chemotherapy had a significantly lower DSS ( $33 \%$ vs $84 \%$ at 5 years, $P<0.001)$ and EFS ( $40 \%$ vs $63 \%$ at 5 years, $P=0.019)$ than patients with an MC of $<20 / 10$ HPF.

Conclusions: The MC and the amount of VT after neoadjuvant therapy for primary, localised, high-grade STS appear to correlate with prognosis. If these results are validated prospectively, then they could provide a rational for the design of neoadjuvant treatment modification/escalation studies, analogue to the EURAMOS-1 trial for bone sarcomas.

The treatment of high-grade soft tissue sarcomas can be challenging (Clarkson and Ferguson, 2004), and neoadjuvant modalities are often implemented to improve tumour resectability and disease outcome (Eilber et al, 2001). Preoperative systemic chemotherapy has been shown to be effective in downstaging large, high-grade tumours
(Demetri et al, 2010) and can be considered for tumours that can only be resected by means of ablative or mutilating surgery (ESMO/ European Sarcoma Network Working Group, 2012).

The assessment of tumour response to neoadjuvant treatment is an important tool in multidisciplinary treatment plans, as it helps

*Correspondence: Dr D Andreou; E-mail: dimosthenis.andreou@ukmuenster.de

Received 31 August 2014; revised 1 November 2014; accepted 1 December 2014; published online 23 December 2014

(c) 2015 Cancer Research UK. All rights reserved 0007-0920/15 
identify patients who might benefit from treatment modification or intensification (Schmidt et al, 1993; Eilber et al, 2001), potential candidates for experimental treatment options (Picci et al, 1993), as well as patients who can be spared the morbidity of continuing an ineffective treatment (Pezzi et al, 1990). Contrary to bone sarcomas, where the histological evaluation of treatment-induced necrosis has been validated as a very important prognostic factor (Picci et al, 1993; Bielack et al, 2002), there is no standardised approach for the histological response assessment of soft tissue sarcomas to neoadjuvant chemotherapy (Lucas et al, 2008). The most commonly used parameter has been tumour necrosis (Huth et al, 1985; Pezzi et al, 1990; Schmidt et al, 1993; Eilber et al, 2001; Menendez et al, 2007; Lucas et al, 2008; Novais et al, 2010) analogue to bone sarcomas. However, the definition of 'good' and 'poor' responders in published studies has not been uniform and the results have been conflicting, with some studies finding that tumour necrosis after neoadjuvant treatment is a good predictor of prognosis (Huth et al, 1985; Pezzi et al, 1990; Eilber et al, 2001; Novais et al, 2010), and other studies claiming that this is not the case (Menendez et al, 2007; Lucas et al, 2008). Although changes in mitotic count (MC), an important prognostic factor in soft tissue sarcomas (Trojani et al, 1984; Tsujimoto et al, 1988), have been described after induction chemotherapy (Coindre et al, 1985), the prognostic value of this parameter in assessing tumour response has been largely ignored.

The objective of this study was to examine whether MC and the amount of viable tumour (VT) following neoadjuvant treatment for primary, localised, high-grade soft tissue sarcoma correlate with prognosis.

\section{PATIENTS AND METHODS}

Study design. Between 2001 and 2011, 58 consecutive patients with primary, locally advanced, non-metastatic, high-grade soft tissue sarcomas underwent neoadjuvant systemic chemotherapy followed by the surgical tumour resection at the sarcoma center Berlin-Brandenburg. One patient underwent induction chemotherapy followed by neoadjuvant isolated limb perfusion before surgical treatment and was excluded from our analysis leaving 57 patients as the subject of this study. All patients signed an informed consent form at hospital admission allowing the use of anonymised data for research purposes.

Data regarding patient demographics, tumour characteristics, first-line treatment, and follow-up were collected prospectively and entered into an electronic database. Further details regarding VT and MC before and after treatment were collected retrospectively from pathology reports. The tumours of patients with missing data were reexamined by a sarcoma pathologist (MW) who was blinded to the clinical characteristics and patients' outcome. Survival analysis was based on follow-up data as of May 2013. Follow-up information for patients who had stopped presenting at our outpatient clinic was gathered by contacting the referring physicians.

Neoadjuvant treatment. The decisions regarding neoadjuvant treatment were made by an interdisciplinary panel, based on tumour histology, localisation, and extent, as well as patient performance status and preference. Systemic chemotherapy protocols consistently involved the combination of an anthracycline (doxorubicin or epirubicin) and an alkylating agent (ifosfamide or dacarbazine). Some patients also received etoposide, while three young patients with pediatric-type sarcomas were additionally treated with vincristine and dactinomycin. Patients were treated with four to six cycles of induction chemotherapy; surgical resection was performed 3 weeks after the last cycle.
Histopathology. All surgical specimens were evaluated by pathologists specialising in bone and soft tissue sarcomas in a standardised manner, according to the technique established for bone sarcomas (Salzer-Kuntschik et al, 1983). Following fixation in a $4 \%$ formalin solution for $24 \mathrm{~h}$, the surgical specimens were bisected along their greatest diameter. A longitudinal section was then obtained, followed by several transversal sections. A gross estimation of the residual amount of VT was recorded, taking into consideration areas of cystic degeneration, haemorrhage, or fibrosis. An amount of $<10 \%$ residual VT was classified as 'low', while an amount of $\geqslant 10 \%$ residual VT was classified as 'high', analogue to the Salzer-Kuntschik grading system for osteosarcoma patients (Salzer-Kuntschik et al, 1983). The number of mitoses was recorded both in the surgical specimen after neoadjuvant treatment and, when available, in the biopsy specimen. Mitoses were counted in at least 10 high power fields (HPF), which correspond to $3.06 \mathrm{~mm}^{2}$ in our microscopes. An MC of $0-9$ mitoses per $10 \mathrm{HPF}$ was classified as 'low', an MC of 10-19 mitoses per $10 \mathrm{HPF}$ as 'intermediate', while an MC of $\geqslant 20$ mitoses per $10 \mathrm{HPF}$ was classified as 'high' (Trojani et al, 1984).

Statistical analysis. Non-parametric analyses were carried out with the Mann-Whitney $U$ and the Wilcoxon signed-rank test. The duration of follow-up and the time to event (local recurrence, metastasis, or disease-related death) were calculated from the date of diagnostic biopsy. Survival curves were calculated with the Kaplan-Meier method (Kaplan and Maier, 1958) and compared with the log-rank test (Mantel, 1966). Statistical analyses were performed with the IBM SPSS Statistics for Windows software version 20.0 (IBM Corp., Armonk, NY, USA). All $P$-values are twosided; a $P$-value $<0.05$ was considered significant.

\section{RESULTS}

Patient demographics, tumour characteristics and neoadjuvant treatment are listed in Table 1. The median patient age at diagnosis was 55 years (range, 16-73 years). The median tumour size at diagnosis, available for 53 patients, was $12 \mathrm{~cm}$ (range, 4-27 cm), while the median tumour size after neoadjuvant treatment was available for 54 patients and amounted to $9 \mathrm{~cm}$ (range, $0-26 \mathrm{~cm}$; $P=0.043$ ). The median follow-up was 44 months for all patients (range, 5-134 months) and 55 months for survivors (range, 13-134 months).

All patients underwent surgical resection of the primary tumour following neoadjuvant treatment. Limb-sparing surgery was performed in 45 of the patients with tumour in the extremities or pelvis, while an amputation was necessary in 8 patients. Surgical margins were clear in 50 patients and microscopically positive in 7 patients. Thirty-five patients underwent adjuvant radiation treatment.

The MC before neoadjuvant treatment was available for 32 patients and amounted to a median of 22/10 HPF (range, 0-150/10 HPF; mean, 32/10 HPF). The MC after neoadjuvant treatment, available for all patients, was significantly lower (median, 10/10 HPF; range, 0-120/10 HPF; mean, 17/10 HPF; $P=0.001$ ). The amount of VT after neoadjuvant treatment was available for 56 patients. Eighteen patients had $<10 \%$ VT, while 38 patients had $\geqslant 10 \%$ VT.

Six patients developed a local recurrence after a median time of 18 months (range, 10-25 months), five of which had clear margins at the surgical treatment of the primary tumour. Distant metastases were diagnosed in 20 patients after a median time of 15 months (range, 7-70 months). At the time of last follow-up, 36 patients were alive without evidence of disease, 3 patients were alive with disease, 15 patients had died of their disease, and 3 patients had died of other causes. Disease-specific survival (DSS) probability at 2 and 5 years amounted to $89 \%$ and $70 \%$, respectively. Event-free 
Table 1. Patient demographics and tumour characteristics

\begin{tabular}{|c|c|c|}
\hline Variable & $n$ & $\%$ \\
\hline Eligible patients & 57 & 100 \\
\hline \multicolumn{3}{|l|}{ Sex } \\
\hline Male & 29 & 51 \\
\hline Female & 28 & 49 \\
\hline \multicolumn{3}{|l|}{ Primary tumour site } \\
\hline Lower extremity & 46 & 81 \\
\hline Upper extremity & 5 & 9 \\
\hline Thoracic wall & 4 & 7 \\
\hline Pelvis & 2 & 3 \\
\hline \multicolumn{3}{|l|}{ Histology } \\
\hline Undifferentiated sarcoma & 24 & 42 \\
\hline Synovial sarcoma & 10 & 17 \\
\hline Leiomyosarcoma & 6 & 11 \\
\hline Rhabdomyosarcoma & 5 & 9 \\
\hline Myxofibrosarcoma & 5 & 9 \\
\hline Dedifferentiated liposarcoma & 4 & 7 \\
\hline Myxoid liposarcoma & 2 & 3 \\
\hline MPNST & 1 & 2 \\
\hline \multicolumn{3}{|l|}{ Tumour grade (FNCLCC) } \\
\hline 2 & 11 & 19 \\
\hline 3 & 46 & 81 \\
\hline \multicolumn{3}{|l|}{ Stage (AJCC) } \\
\hline Ila & 9 & 16 \\
\hline III & 48 & 84 \\
\hline \multicolumn{3}{|c|}{$\begin{array}{l}\text { Abbreviations: } \mathrm{AJCC}=\text { American Joint Committee on Cancer; FNCLCC = Fédération } \\
\text { Nationale des Centres de Lutte Contre le Cancer; MPNST = malignant peripheral nerve } \\
\text { sheath tumour. }\end{array}$} \\
\hline
\end{tabular}

survival (EFS) probability at 2 and 5 years was $64 \%$ and $57 \%$, respectively.

The amount of VT after systemic chemotherapy was significantly associated with DSS and EFS (Figure 1). Patients with $<10 \%$ VT had a DSS of $94 \%$ at 5 years, compared to $61 \%$ for patients with $\geqslant 10 \%$ VT $(P=0.033)$, while EFS amounted to $75 \%$ compared to $48 \%(P=0.030)$. The MC before neoadjuvant treatment had no statistically significant influence on DSS or EFS in this selected group of patients with high-grade tumours (Table 2). On the other hand, patients with a high MC after systemic chemotherapy had a significantly lower DFS $(P<0.001)$ and EFS $(P=0.019)$ than patients with a low and intermediate MC (Figure 2; Table 2).

To evaluate whether a more accurate prediction of prognosis could be achieved, we examined the combined influence of MC after treatment and VT on survival. Patients with an MC of $<20$ / $10 \mathrm{HPF}$ and a VT of $<10 \%$ had a strong trend for an improved DSS $(100 \%$ vs $76 \%$ at 5 years, $P=0.068)$ and an improved EFS (77\% vs 54\% at 5 years, $P=0.083$ ), compared with patients with an MC of $<20 / 10 \mathrm{HPF}$ and a VT of $\geqslant 10 \%$ (Figure 3 ).

Finally, the MC after neoadjuvant treatment had a significant influence on post-relapse survival (PRS) probability. Following a local or systemic recurrence, patients with an MC of $<20 / 10 \mathrm{HPF}$ had a PRS of $70 \%$ at 2 years, compared to $22 \%$ for patients with an MC of $\geqslant 20 / 10 \mathrm{HPF}(P=0.003)$ (Figure 4$)$. With the amount of patients available for this study, the amount of VT after chemotherapy did not correlate with PRS. Patients with $<10 \%$ VT had a PRS of $75 \%$ at 2 years, compared to $49 \%$ for patients with $\geqslant 10 \%$ VT $(P=0.423)$.

\section{DISCUSSION}

The histological assessment of tumour response to neoadjuvant chemotherapy is one of the most important prognostic factors in
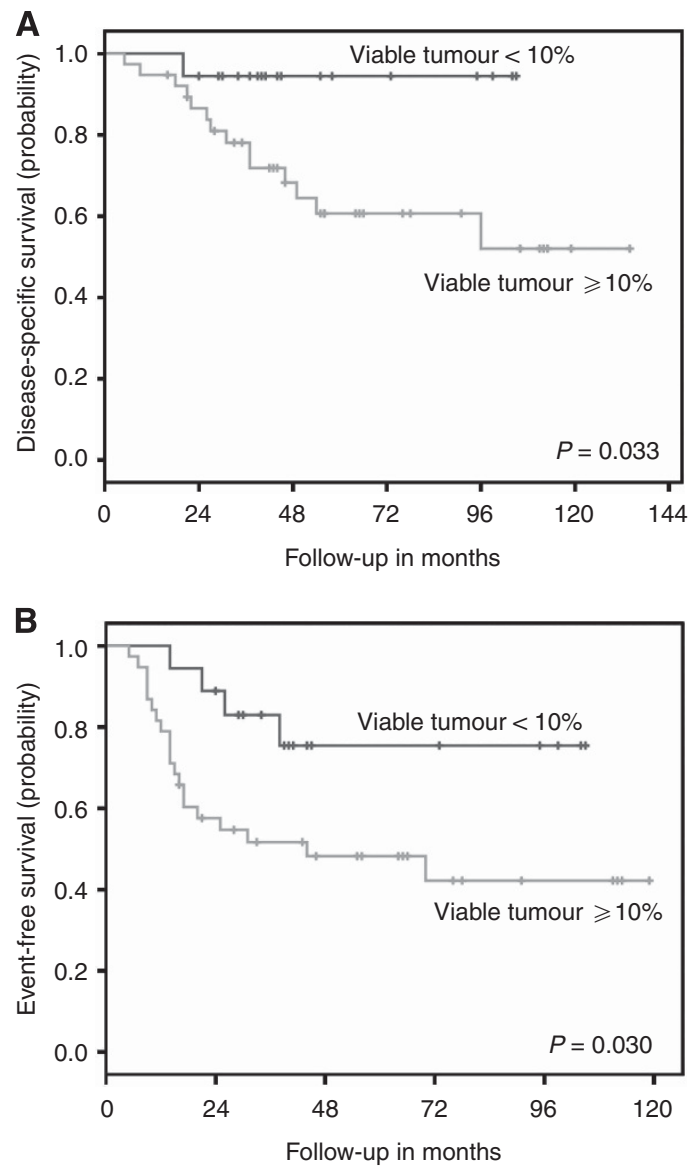

Figure 1. Disease-specific (A) and event-free survival (B) probability according to the amount of VT after treatment.

osteosarcoma (Bielack et al, 2002) and Ewing sarcoma (Picci et al, 1993). While several studies have sought to determine the prognostic value of histological response assessment in soft tissue sarcomas (Huth et al, 1985; Pezzi et al, 1990; Schmidt et al, 1993; Eilber et al, 2001; Menendez et al, 2007; Lucas et al, 2008), the definitions of 'good' and 'poor' response were varied and the results conflicting. Our study aimed at shedding new light on this issue, evaluating the impact of not only tumour necrosis but also $\mathrm{MC}$, a factor that has been largely overlooked in previous analyses. Inevitably, the differences in systemic chemotherapy protocols used over the years caused for some inhomogeneity in our patient cohort, which is one of the limitations of this study, while another is its retrospective nature. Nevertheless, all patients received at least an anthracycline and an alkylating agent, both of which are regarded as active and are recommended for first-line treatment in most soft tissue sarcoma subtypes (Demetri et al, 2010; ESMO/ European Sarcoma Network Working Group, 2012).

A further limitation of our study regards the lack of a validated technique to assess the response of soft tissue sarcomas to neoadjuvant treatment. As a result, many authors use techniques developed for and validated in osteosarcoma patients instead (Huth et al, 1985; Menendez et al, 2007). This approach has its drawbacks, as reductions in tumour volume under neoadjuvant chemotherapy are more common in soft tissue sarcoma than in osteosarcoma patients. These volume reductions can lead to a discrepancy between clinical and histopathological response - for example, a given tumour might reduce significantly in size during neoadjuvant treatment but show mostly viable areas in histopathology (Lucas et al, 2008). Due to these drawbacks, we chose to also evaluate the prognostic significance of the MC after treatment as a marker of histopathological tumour response to treatment. 
Table 2. Mitotic count and survival

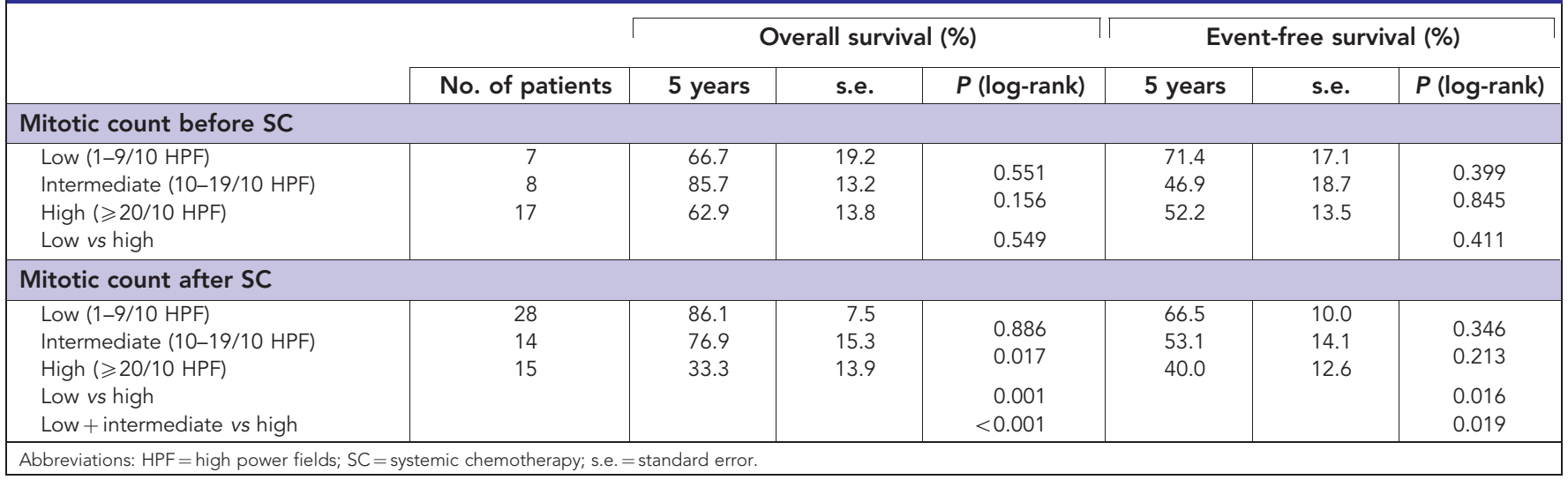
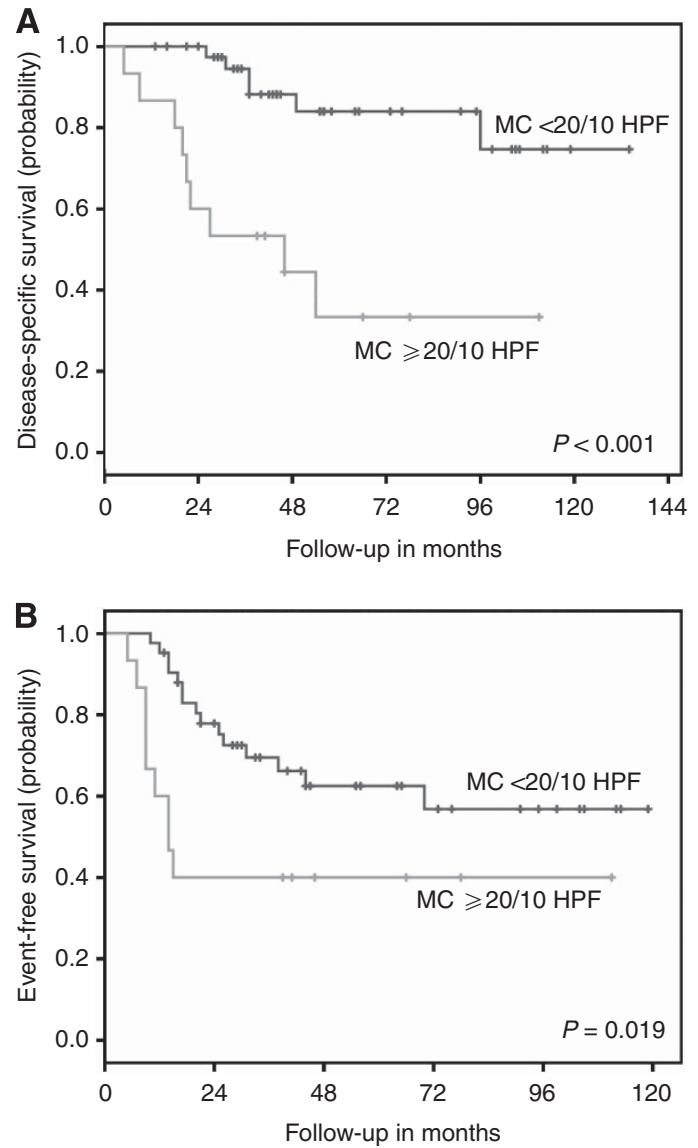

Figure 2. Disease-specific (A) and event-free survival (B) probability according to the $\mathrm{MC}$ after treatment.

The amount of VT after systemic chemotherapy with a cutoff at $10 \%$ had a significant correlation to DSS and EFS. Our results are in accordance with those of several previous studies (Huth et al, 1985; Pezzi et al, 1990; Eilber et al, 2001; Novais et al, 2010), however some authors have reported a lack of association between the amount of VT and survival (Menendez et al, 2007; Lucas et al, 2008). One possible explanation for these conflicting findings might be the fact that the neoadjuvant treatment protocols used in the aforementioned studies greatly varied in terms of the chemotherapy agents that were applied, their dosage, as well as in the use of neoadjuvant radiation treatment and adjuvant chemotherapy.
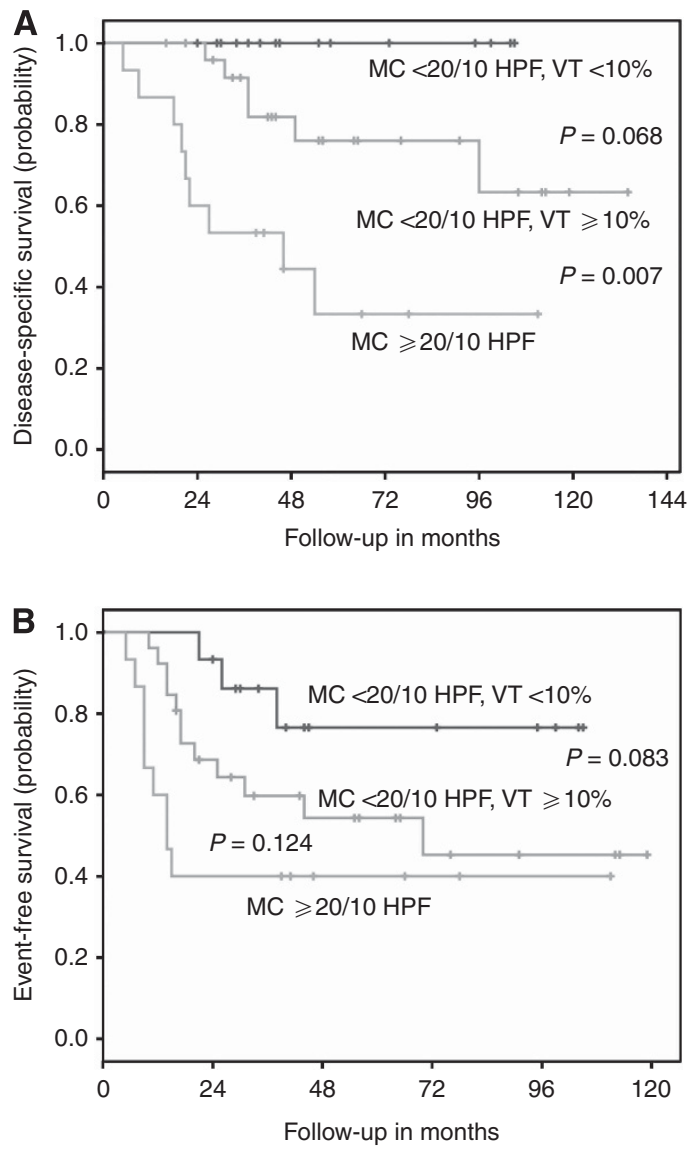

Figure 3. Disease-specific (A) and event-free survival (B) probability according to the MC and the amount of VT.

While in this selected group of patients the MC before treatment had no influence on DSS or EFS, the most important predictor of survival was the MC after neoadjuvant treatment. To our knowledge, this is the first time this aspect of tumour response has been evaluated in detail in soft tissue sarcomas following systemic chemotherapy. Whether the histological response to neoadjuvant chemotherapy is a marker of the tumour's susceptibility to preoperative treatment or an indication of the tumour's biologic aggressiveness, as has been hypothesised in the past (Huth et al, 1985), remains unclear. However, the information provided can still be used to identify a group of high-risk patients who might benefit from second-line or experimental treatments (Pezzi et al, 1990). 


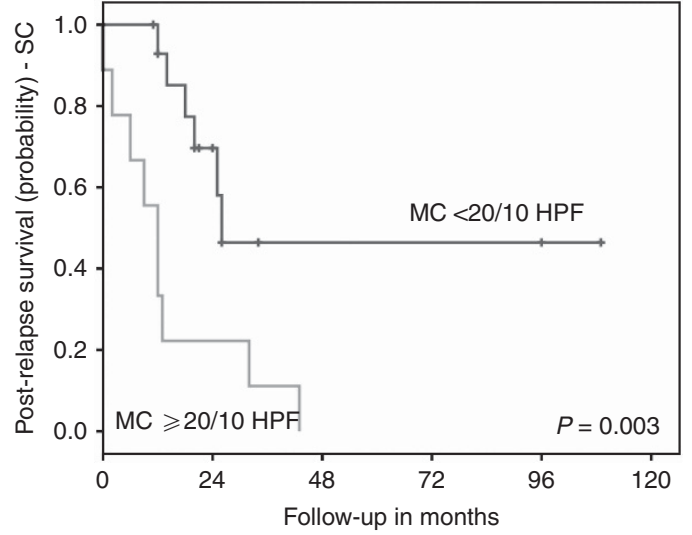

Figure 4. Post-relapse survival probability according to the MC after chemotherapy.

An obvious disadvantage of using the MC after neoadjuvant treatment as a prognostic factor is that it is subject to interobserver variation and sensitive to delays in fixation (Daugaard et al, 1993). Ki-67 staining has been proposed as an alternative method to measure cell proliferation (Daugaard et al, 1993), as it has been shown to have a somewhat higher reproducibility than MC (Hasegawa et al, 2002). Nevertheless, MC remains one of the parameters used in the most widely employed system to determine tumour grade in soft tissue sarcomas (Deyrup and Weiss, 2006) and has also been established in gastrointestinal stromal tumours (GIST) as one of the three prognostic factors used to identify patients at a high risk for recurrence who should be considered for adjuvant treatment (Joensuu et al, 2012).

The combined evaluation of the amount of VT and the MC after chemotherapy appears to separate patients into three prognostic groups (Figure 3). If these results are confirmed in a separate patient cohort, then they could provide a rationale for the design of neoadjuvant treatment modification/escalation studies, analogue to the EURAMOS-1 (Marina et al, 2009) and EURO-B.O.S.S. (Carrle and Bielack, 2006) trials for bone sarcomas. It should be noted that, contrary to osteosarcoma and Ewing sarcoma, neoadjuvant chemotherapy is not the standard of care in soft tissue sarcoma. However, the guidelines of the European Society for Medical Oncology (ESMO) recommend the use of neoadjuvant chemotherapy for the treatment of non-resectable tumours or tumours amenable only to mutilating surgery (ESMO/European Sarcoma Network Working Group, 2012). Furthermore, it has been shown that neoadjuvant chemotherapy may be associated with an improved DSS in patients with large $(>10 \mathrm{~cm})$, high-grade soft tissue sarcomas (Grobmyer et al, 2004).

In conclusion, the MC and the amount of VT after neoadjuvant therapy for primary, localised, high-grade soft tissue sarcoma appear to correlate with prognosis. Obviously, these findings need to be validated in larger, homogeneous studies. As the focus of systemic chemotherapy trials has been shifting to tailored studies for patients with different soft tissue sarcoma subtypes in recent years (Eriksson, 2010), we believe that this provides an excellent opportunity to prospectively examine the prognostic relevance of the $\mathrm{MC}$ and the amount of VT after treatment in different subgroups of soft tissue sarcoma patients.

\section{CONFLICT OF INTEREST}

The authors declare no conflict of interest.
REFERENCES

Bielack SS, Kempf-Bielack B, Delling G, Exner GU, Flege S, Helmke K, Kotz R, Salzer-Kuntschik M, Werner M, Winkelmann W, Zoubek A, Jurgens H, Winkler K (2002) Prognostic factors in high-grade osteosarcoma of the extremities or trunk: an analysis of 1,702 patients treated on neoadjuvant cooperative osteosarcoma study group protocols. J Clin Oncol 20(3): 776-790.

Carrle D, Bielack SS (2006) Current strategies of chemotherapy in osteosarcoma. Int Orthop 30(6): 445-451.

Clarkson P, Ferguson PC (2004) Primary multidisciplinary management of extremity soft tissue sarcomas. CurrTreat Opt Oncol 5(6): 451-462.

Coindre JM, Nguyen BB, Goussot JF, de Mascarel I, Maree D, de Mascarel A, Trojani M (1985) [Histological changes after chemotherapy of soft tissue sarcomas in the adult]. Ann Pathol 5(2): 95-99.

Daugaard S, von Glabbeke M, Schiodt T, Mouridsen HT (1993) Histopathological grade and response to chemotherapy in advanced soft tissue sarcomas. Eur J Cancer 29A(6): 811-813.

Demetri GD, Antonia S, Benjamin RS, Bui MM, Casper ES, Conrad 3rd EU, DeLaney TF, Ganjoo KN, Heslin MJ, Hutchinson RJ, Kane 3rd JM, Letson GD, McGarry SV, O’Donnell RJ, Paz IB, Pfeifer JD, Pollock RE, Randall RL, Riedel RF, Schupak KD, Schwartz HS, Thornton K, von Mehren M, Wayne J (2010) Soft tissue sarcoma. J Natl Compr Canc Netw 8(6): 630-674.

Deyrup AT, Weiss SW (2006) Grading of soft tissue sarcomas: the challenge of providing precise information in an imprecise world. Histopathology 48(1): 42-50.

Eilber FC, Rosen G, Eckardt J, Forscher C, Nelson SD, Selch M, Dorey F, Eilber FR (2001) Treatment-induced pathologic necrosis: a predictor of local recurrence and survival in patients receiving neoadjuvant therapy for high-grade extremity soft tissue sarcomas. J Clin Oncol 19(13): 3203-3209.

Eriksson M (2010) Histology-driven chemotherapy of soft-tissue sarcoma. Ann Oncol 21(Suppl 7): vii270-vii276.

ESMO/European Sarcoma Network Working Group (2012) Soft tissue and visceral sarcomas: ESMO Clinical Practice Guidelines for diagnosis, treatment and follow-up. Ann Oncol 23(Suppl 7): vii92-vii99.

Grobmyer SR, Maki RG, Demetri GD, Mazumdar M, Riedel E, Brennan MF, Singer S (2004) Neo-adjuvant chemotherapy for primary high-grade extremity soft tissue sarcoma. Ann Oncol 15(11): 1667-1672.

Hasegawa T, Yamamoto S, Nojima T, Hirose T, Nikaido T, Yamashiro K, Matsuno Y (2002) Validity and reproducibility of histologic diagnosis and grading for adult soft-tissue sarcomas. Hum Pathol 33(1): 111-115.

Huth JF, Mirra JJ, Eilber FR (1985) Assessment of in vivo response to preoperative chemotherapy and radiation therapy as a predictor of survival in patients with soft-tissue sarcoma. Am J Clin Oncol 8(6): 497-503.

Joensuu H, Vehtari A, Riihimaki J, Nishida T, Steigen SE, Brabec P, Plank L, Nilsson B, Cirilli C, Braconi C, Bordoni A, Magnusson MK, Linke Z, Sufliarsky J, Federico M, Jonasson JG, Dei Tos AP, Rutkowski P (2012) Risk of recurrence of gastrointestinal stromal tumour after surgery: an analysis of pooled population-based cohorts. Lancet Oncol 13(3): 265-274.

Kaplan E, Maier P (1958) Nonparametric estimation from incomplete observations. J Am Stat Assoc 53: 457-481.

Lucas DR, Kshirsagar MP, Biermann JS, Hamre MR, Thomas DG, Schuetze SM, Baker LH (2008) Histologic alterations from neoadjuvant chemotherapy in high-grade extremity soft tissue sarcoma: clinicopathological correlation. Oncologist 13(4): 451-458.

Mantel N (1966) Evaluation of survival data and two new rank order statistics arising in its consideration. Cancer Chemother Rep 50(3): 163-170.

Marina N, Bielack S, Whelan J, Smeland S, Krailo M, Sydes MR, Butterfass-Bahloul T, Calaminus G, Bernstein M (2009) International collaboration is feasible in trials for rare conditions: the EURAMOS experience. Cancer Treat Res 152: 339-353.

Menendez LR, Ahlmann ER, Savage K, Cluck M, Fedenko AN (2007) Tumor necrosis has no prognostic value in neoadjuvant chemotherapy for soft tissue sarcoma. Clin Orthop Relat Res 455: 219-224.

Novais EN, Demiralp B, Alderete J, Larson MC, Rose PS, Sim FH (2010) Do surgical margin and local recurrence influence survival in soft tissue sarcomas? Clin Orthop Relat Res 468(11): 3003-3011.

Pezzi CM, Pollock RE, Evans HL, Lorigan JG, Pezzi TA, Benjamin RS, Romsdahl MM (1990) Preoperative chemotherapy for soft-tissue sarcomas of the extremities. Ann Surg 211(4): 476-481. 
Picci P, Rougraff BT, Bacci G, Neff JR, Sangiorgi L, Cazzola A, Baldini N, Ferrari S, Mercuri M, Ruggieri P et al (1993) Prognostic significance of histopathologic response to chemotherapy in nonmetastatic Ewing's sarcoma of the extremities. J Clin Oncol 11(9): 1763-1769.

Salzer-Kuntschik M, Delling G, Beron G, Sigmund R (1983) Morphological grades of regression in osteosarcoma after polychemotherapy - study COSS 80. J Cancer Res Clin Oncol 106(Suppl): 21-24.

Schmidt RA, Conrad 3rd EU, Collins C, Rabinovitch P, Finney A (1993) Measurement and prediction of the short-term response of soft tissue sarcomas to chemotherapy. Cancer 72(9): 2593-2601.

Trojani M, Contesso G, Coindre JM, Rouesse J, Bui NB, de Mascarel A, Goussot JF, David M, Bonichon F, Lagarde C (1984) Soft-tissue sarcomas of adults; study of pathological prognostic variables and definition of a histopathological grading system. Int J Cancer 33(1): 37-42.

Tsujimoto M, Aozasa K, Ueda T, Morimura Y, Komatsubara Y, Doi T (1988) Multivariate analysis for histologic prognostic factors in soft tissue sarcomas. Cancer 62(5): 994-998.

This work is published under the standard license to publish agreement. After 12 months the work will become freely available and the license terms will switch to a Creative Commons AttributionNonCommercial-Share Alike 3.0 Unported License. 